\title{
Topology Control and Routing in Large Scale Wireless Sensor Networks
}

\author{
Ines Slama, Badii Jouaber, Djamal Zeghlache \\ Telecom and Management SudParis, Evry, France \\ E-mail: \{Ines.slama, Badii.Jouaber, Djamal.Zeghlache\}@it-sudparis.eu \\ Received May 20, 2010; revised May 28, 2010; accepted June 5, 2010
}

\begin{abstract}
In this paper, a two-tiered Wireless Sensor Network (WSN) where nodes are divided into clusters and nodes forward data to base stations through cluster heads is considered. To maximize the network lifetime, two energy efficient approaches are investigated. We first propose an approach that optimally locates the base stations within the network so that the distance between each cluster head and its closest base station is decreased. Then, a routing technique is developed to arrange the communication between cluster heads toward the base stations in order to guaranty that the gathered information effectively and efficiently reach the application. The overall dynamic framework that combines the above two schemes is described and evaluated. The experimental performance evaluation demonstrates the efficacy of topology control as a vital process to maximize the network lifetime of WSNs.
\end{abstract}

Keywords: Wireless Sensor Networks, Routing, Topology Control, Base Stations Placement, Energy Efficiency

\section{Introduction}

Achieving maximum lifetime in stationary WSNs by optimally using the energy within sensor nodes has been the subject of significant researches in the last recent years. In this field, radio transmission and reception operations are being identified as one of the most energy consuming features.

On the other hand, the development of large-scale sensor networks has drawn a lot of attention. Indeed, according to the application, the number of sensor nodes deployed to sense a specific phenomenon may be in the order of hundreds or thousands and can reach a value of millions. Therefore, the large size of wireless sensor networks inevitably introduces significant scalability concerns. One of the main challenges is then to set up new architectures and mechanisms that can efficiently scale up with the growing number of nodes that may be required to ensure adequate coverage of large areas of interest. At the same time, these new architectures and mechanisms should maintain low energy consumption per node so as to get by with energy guaranty acceptable network lifetime. The evaluation of the scalability of an algorithm or protocol is mainly based on a well-known metric for WSNs, which is the network lifetime. The objective is to avoid significant degradations of the network lifetime when the number of nodes composing the WSN increases.

One promising approach to solve the problem of scalability is to build hierarchies among the nodes, such that the topology of the network is abstracted [1]. Indeed, flat topologies are difficult to scale up since communications between thousands or perhaps millions of nodes in a ad hoc fashion lead to degraded performances and hence higher energy consumption.

Hierarchical topologies are rather recommended in such scenarios. The network protocols or algorithms designed for these architectures are generally highly scalable. Moreover, such architecture enables better resources allocation and improves power control over the network [2].

Such architectures are consisted of sensor clusters and one or more base stations. Each cluster is managed by a cluster head. Cluster members are generally small sensor nodes that capture relevant information from the area of interest and send it to their cluster head. This latter creates from the received data a comprehensive local view that is then sent to a base station (or sink). By combining all the data received from the different cluster heads, the base stations generate a comprehensive global view for the entire WSN coverage that it finally sent to the appli- 
cation level. In practice, cluster members do not communicate with each others whereas cluster heads can be involved in inter cluster heads relaying. Both cluster members and cluster heads are battery powered and energy constrained. However, cluster heads are considered to have more computing and storage capacities than member nodes. Base stations can be mobile or located in flexible positions and have infinite processing, storage and power resources. In such scenario, if a sensing node (cluster member) is drained out of energy, the cluster head may still be able to provide a comprehensive local-view by data generated by other sensor nodes in the cluster. However, if a cluster head runs out of energy, the whole cluster coverage is broken. More than that, the relaying task performed by this cluster head from/to neighboring cluster heads toward the sink is also lost. This may affect routes and lead in some cases to the disconnection of an entire region of the network, which may be dramatic for the network mission. Therefore, we are more concerned about the energy constraint of cluster heads.

Although cluster heads can have better initial energy provisioning than sensor nodes, cluster heads also consume energy at a considerably higher rate due to the transmission of gathered data over much longer distances [3]. Moreover, cluster heads relay not only data generated within their own clusters but also data they may receive from their cluster head neighbors. Cluster Heads also consume considerable energy for managing their clusters and gathering information from their cluster members (reception operations, data aggregation, signaling, etc). Cluster heads perform then two high energy cost roles. The first is related to forwarding and relaying tasks and the second concerns their own cluster management. To increase the network lifetime, the communication between the cluster heads should be managed in an energy efficient manner. To this end, an energy aware routing scheme should be investigated to fairly balance the energy consumption among the cluster heads according to both their available amount of energy and their role in the network and then route around cluster heads that are dying or need higher energy to manage their clusters.

On the other hand, base stations have infinite processing, storage and power resources. Therefore, an efficient usage of these flexible and mobile base stations to increase the network lifetime should be investigated especially when the sensing nodes and cluster heads are stationary or have very low mobility. The idea behind this is to decrease the distance between each cluster head and the nearest base station. In fact, when a higher number of base stations are distributed within the WSN, the paths length from any cluster head to its nearest base station is decreased leading to lower energy consumption and therefore to higher network lifetime. Moreover, since multi hop communication is used between cluster heads, the ones that are one hop from the base station drain their energy faster than other nodes because they have to relay messages originated from many other nodes (Cluster Heads) in addition to delivering their own messages. Therefore, using mobile base stations may help distributing the energy consumption over the different relaying cluster heads and then increase the network lifetime. Base stations trajectories can be rather controlled by the application or follow a specific mobility model in which case an estimation of their locations can be computed like in [4].

Motivated by these issues, we focus, in this paper, on first, how to optimally locate the base stations in the network and second, on how to arrange the communication between cluster heads toward the base stations, both in order to guaranty that the gathered information effectively and efficiently reach the application. This is generally referred to as topology control. Our goal is to maximize the lifetime of a large-scale and energy constrained WSN.

The remainder of this paper is organized as follows. A related work is presented in Section 2. In Section 3, we give the system architecture of a two-tiered WSN, its Cluster Heads energy dissipation model, and the definition of the whole network lifetime. In Section 4, we present the approach that optimally locates multiple mobile base stations in the network such that the energy consumption is fairly distributed over the different Cluster Heads. In Section 5, by extending an optimal routing approach presented in a previous work [5], we introduce a new optimization scheme that arranges the multi-hop communication between the Cluster Heads while considering their residual amount of energy and the role they play in the network leading to a maximum network lifetime. This new optimal routing scheme takes into account the energy required by each Cluster Head to gather data from its cluster as well as to relay the packets coming from its Cluster Head neighbors toward the corresponding base station. We describe then in section 6 the overall dynamic framework. Simulations conducted to evaluate this global approach are described and commented in section 7. Section 8 concludes this paper and points out the directions for future work.

\section{Prior Work}

To solve energy constrained problem in wireless sensor networks field, many routing protocols have been proposed; especially, cluster based routing protocols have many advantages such as reducing control messages, maximizing bandwidth reusability, enhanced resource allocation, larger scalability and improved power control.

LEACH (Low-Energy Adaptive Clustering Hierarchy) proposed in [6] includes a distributed cluster formation technique that enables self-organization of large numbers 
of nodes and rotating cluster head positions, so that the high energy dissipation in communicating with the base station is spread over all sensor nodes in the sensor network. However, LEACH can suffer from the clustering overhead that may result in supplementary power consumption. Moreover, cluster head rotation requires that all the nodes be capable of performing data aggregation, cluster management and routing decisions. This results in extra hardware complexity in all the nodes.

In contrast, in [7], the above complexity is embedded in only a few nodes (cluster head nodes). In [7], authors aim to obtain the minimum number of sensing nodes, cluster heads, and battery energy to ensure at least $\mathrm{T}$ unit of lifetime. Nodes were divided into two categories: nodes 0 are sensing node and nodes 1 are cluster heads. Analysis results show that the number of cluster heads should be of the order of square root of the number of sensing nodes. However, authors don't give any exact evaluation of the maximum lifetime of the network. Moreover, it was assumed in this work that cluster heads directly communicate (i.e. one hop communication) with the base station, which is difficult to be applied to realistic scenarios. It has also been observed that the nodes close to the cluster heads have high-energy consumption due to packet relaying operations. But, one of the most significant observation is the sharp cutoff effect, to maximize the lifetime does not hold at all time.

In [3], a two-tiered wireless sensing network was considered and the authors observed the property that the first tier nodes are important for the lifetime of the entire network. They investigated a topology control approach to maximize the topological lifetime of the network in terms of the base station placement for the two-tiered sensor networks where the nodes are deployed within clusters. However, the optimal base station locations were obtained without considering the inter-cluster heads relaying. Then, once the base stations located, the inter cluster heads relaying may be not applicable in some cases.

In [8], authors have developed an analytical model that describes the communication load distribution in WSNs and proved that base station mobility is a strategy that deserves to be considered when optimizing the network lifetime. They have further shown that the optimum movement strategy for a mobile base station is to follow the periphery when the deployment area is circular.

Network lifetime elongation using mobile base stations has also been investigated in [9]. The author gave a novel linear programming formulation for the joint problem of determining the movement of the sink and the sojourn time at different points in the network. Simulations have shown that lifetime maximizing solutions are achieved by non-uniform sojourn time distributions among grid points depending on the shape of the deployment area.

In [10], authors propose to divide time into rounds and to dynamically relocate multiple sinks, at different positions along the periphery of the sensed field, at the beginning of each of these rounds. An integer linear program is used to determine the new locations of the different base stations. Results have shown that the energy consumption of individual sensor nodes is better balanced and the overall energy consumption of all sensors is minimized.

In [11], authors propose a different approach to find the optimal locations of multiple stationary sink nodes. The proposed scheme allows sensor nodes to communicate with one or multiple sinks through multiple paths in order to improve the network lifetime.

Another approach to solve the problem of multiple mobile base station placements is proposed in [12]. An electrostatic model is applied to determine sinks' locations and to coordinates the movements of these sinks considering the network state.

Unfortunately, most of the above base stations locations strategies are proposed and evaluated over small to medium size wireless sensor networks (typically less than 100 nodes). For large-scale wireless sensor networks, where hundreds or thousands of nodes can be deployed, the placement of multiple base stations still requires advanced studies. For instance, and as illustrated on Figure 1, if we consider the case where the sinks are located along the periphery as stated in [10], the paths between each node and its nearest sink is relatively short when the number of nodes is limited. However, the more the area size increases and/or the number of nodes within it increases, the longer this path is and the shorter the sensor nodes lifetime will be.

\section{System Model}

\subsection{Network Architecture}

We consider, in this work, a large number of stationary and heterogeneous sensor nodes covering a given area of interest. For scalability management ends, the topology of the network is abstracted and the nodes are organized into a two-tiered WSN as depicted in Figure 2.

It consists of a number of clusters and multiple mobile base stations. Each cluster is composed of a set of Sensing Nodes and one Cluster Head. Sensing Nodes are small, low cost and densely deployed in each cluster. They are responsible of sensing raw data and then forwarding it to their corresponding Cluster Head. We consider that cluster formation is based on neighborhood. Hence, direct transmissions can be used inside each cluster. However, Sensing Nodes do not communicate with other Sensing Nodes in the same or other clusters. Cluster Heads, on the other hand, have much more responsibilities. First, they manage their clusters (send queries, instruct some nodes to be in idle or sleep status...) 


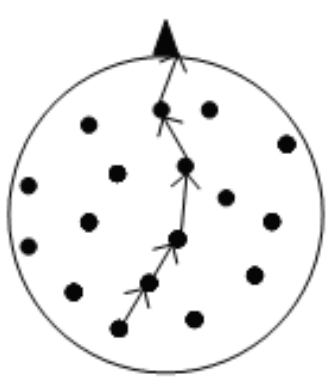

(a)

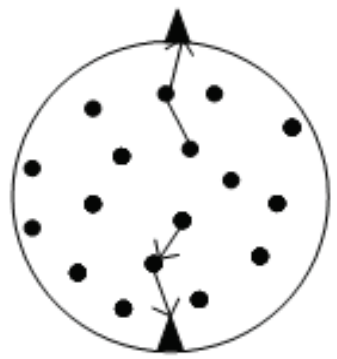

(b)

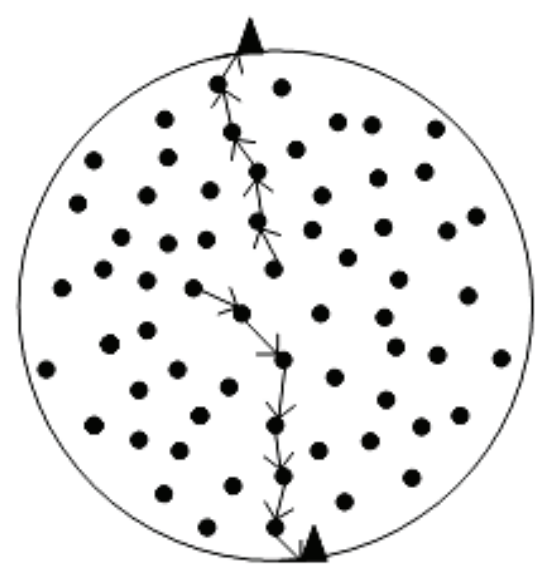

(c)

Figure 1. Multiple base stations placement. (a) a single base station on the periphery on a small scale WSN; (b) two base stations on the periphery on a small scale WSN; (c) two base stations on the periphery on a large scale WSN.

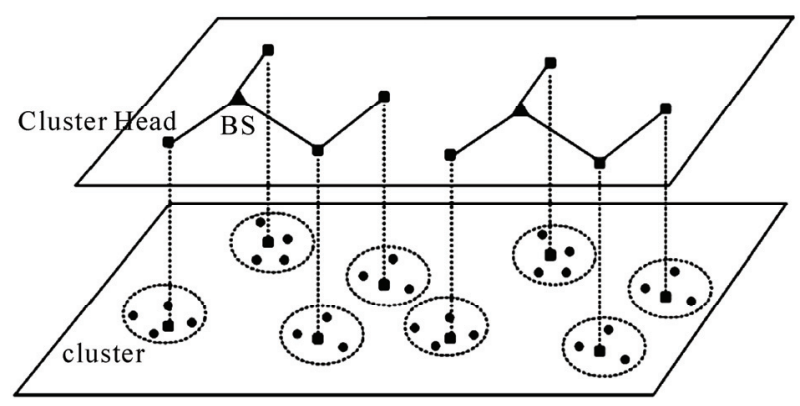

Figure 2. A two-tiered wireless sensor network.

and gather data from their cluster members. Second, they perform aggregation of this data to eliminate redundancy and minimize the number of transmissions and thus save energy. The aggregated data at each Cluster Head represents a local view of its cluster. Third, they transmit the composite bit-stream towards the nearest base station. Each base station can then generate a comprehensive global view of the entire network coverage by combining the different local view data received from the different Cluster Heads [3].

While direct communication is used between Sensing
Nodes and Cluster Heads, it may be inappropriate between Cluster Heads and Base Stations. Indeed, distances between them can be large. In such cases, direct transmissions will require high power consumption. In addition, direct transmissions may be simply impossible since base stations can be out of the range of some Cluster Heads. Multi-hop communication is then used. Consequently, Cluster Heads can also be involved in inter Cluster Head relaying.

Two main roles/responsibilities can be then defined for Cluster Heads. The first is related to their clusters and consists of managing their cluster members and gathering information from them. The second is related to forwarding activities and consists of relaying their own packets as well as packets they may receive from their Cluster Head neighbors towards the corresponding Base Station.

Although, both Sensing Nodes and Cluster Heads are energy constrained, Cluster Heads are considered to have more computing and storage capacities than member nodes. Base Stations, however, have infinite processing, storage and power resources. They can be mobile or located in flexible positions.

Note that initial energy allocation plays an important role in topology control for WSNs. Attributing different initial energy levels to the nodes according to their position and role in the network can significantly improve the network lifetime [3]. However, since fixed initial energy sheme is used in practice (among nodes of the same category, Cluster Heads or Sensing Nodes), we adopt this scheme for the rest of this paper.

In such scenario, Cluster Heads represent the logical bridge between the two tiers of the network architecture: the lower-tier Sensing Nodes and their Cluster Heads and the upper-tier Cluster Heads and Base Stations. They are then vital for the network mission success. Therefore and as stated previously, Cluster heads should be given all our attention in terms of energy efficiency.

Once Cluster Heads and Sensing Nodes are deployed, an immediate challenge is to optimally locate the Base Stations such that the network lifetime is maximized. After Base Stations are located, these latters can compute the inter-Cluster Heads routing scheme. This scheme should instruct Cluster Heads to communicate in an energy efficient manner and fairly distribute the energy consumption among them to achieve a longer network lifetime.

\subsection{Network Graph}

We consider that the network is organized into $N$ Clusters with one Cluster Head for each Cluster. In the rest of this paper, we will note a Cluster $i$ by $C_{i}$ and its corresponding Cluster Head by $\mathrm{CH}_{i}, i=1$ to $N$. All the Sensing Nodes in cluster $C_{i}$ can communicate directly with 
the Cluster Head $\mathrm{CH}_{i}$.

The network is modeled as an undirected connected graph $G(H, A)$ where $H$ is the set of Cluster Heads, $H=\left\{\mathrm{CH}_{i}, i=1\right.$ to $\left.N\right\}$ and $A$ the set of all undirected links $\left(\mathrm{CH}_{i}, \mathrm{CH}_{j}\right)$ where $\mathrm{CH}_{i}, \mathrm{CH}_{j}$ are two Cluster Heads of $H$.

Let $L_{i}$ be the set of Cluster Heads neighbors of Cluster Head $\mathrm{CH}_{i}$. $\mathrm{L}_{i}$ is composed of all Cluster Heads that can be reached by $\mathrm{CH}_{i}$ using a transmission power equal or less than a maximum predefined threshold. All links are assumed to be bidirectional.

\subsection{Energy Model}

In this work, we assume the following radio energy model. Note that $E_{\text {elec }}$ and $\varepsilon_{\text {amp }}$ represent the energy consumed respectively to run the radio electronics and the power amplifier.

Thus, the energy consumed at node $i$ when transmitting information to node $j$ at rate $x_{i j}$ can be written as:

$$
E_{t}\left(x_{i j}, d_{i j}\right)=\left(E_{\text {elec }}+\varepsilon_{a m p} d_{i j}{ }^{2}\right) x_{i j}=e_{i j} x_{i j}
$$

And the energy consumed at node $j$ when receiving information from node $i$ at rate $x_{i j}$ :

$$
E_{r}\left(x_{i j}\right)=E_{\text {elec }} x_{i j}=e_{r} x_{i j}
$$

Moreover, since data aggregation (fusion) is performed within the network, the energy consumed at node $j$ to aggregate information received from node $i$ at rate $x_{i j}$ can be written as:

$$
E_{a}\left(x_{i j}\right)=\gamma x_{i j}=e_{a} x_{i j}
$$

where:

$d_{i j}$ is the Euclid distance between node $i$ and node $j$.

$e_{i j}$ is the transmission energy required to transmit one data unit from node $i$ to node $j$.

$e_{r}$ is the energy required for the reception of one data unit.

$\gamma$ is constant and called the aggregation energy consumption coefficient [1].

$E_{a}$ is the energy required to the fusion of one data unit.

\subsection{Lifetime Definitions}

First, we consider that each cluster in the network dies when no more reliable information can be delivered from the cluster members. A Cluster Head whose cluster is dead, continue performing relaying data from/to neighboring Cluster Heads (i.e., its second role).

Second, we define the lifetime of the whole network as the metric for determining the optimality of the rout- ing algorithm.

We define in this work the lifetime of the whole cluster-based sensor network as the period of time that ends when a first Cluster Head runs out of energy. This implies that every Cluster Head is vital for the application and cannot be substituted by others.

Hence, maximizing the network lifetime can be achieved by delaying as much as possible the first Cluster Head death.

\section{Base Stations Placement}

We propose in this section to enhance Base Station placement in a two-tiered large-scale WSN.

The idea behind this is to efficiently deploy Multiple Mobile Base Stations within the network. Multiple Base Stations are used to decrease the distance between each Cluster Head and its nearest base station. Base stations are also chosen to be mobile to make the set of Cluster Heads close to the Base Stations and then overloaded, change over the time. This should guaranty a fair distribution of the energy consumption among the different Cluster Heads in the network and hence improve the network lifetime. The challenge here is then to define the initial locations and the movement trajectories of the different Base Stations.

Since we deal with a large-scale WSN, an intuitively appropriate solution is to decompose the underlying sensor network and then optimize energy usage in each of the sub-networks independently. The objective is to take advantage of the powerful and efficient Base Station placement techniques proposed for small scale WSNs. In order to apply these techniques over large-scale WSNs, we propose to first divide the network into sub-networks according to specific criteria. An adequate Base Station placement technique can then be applied independently within each of the defined sub-networks.

Graph partitioning is a promising approach to split a large sensor network into balanced sub-networks. In practice, different criteria can be considered in order to partition a large-scale two-tiered wireless sensor network. Since multi-hop communication is used between Cluster Heads toward base stations, one simple objective is to create balanced sub-networks (in terms of number of Cluster Heads/clusters) that group the Cluster Heads according to their neighborhood. This allows creating smaller two-tiered sub-networks with similar characteristics that can be easily optimized, independently but in the same way.

In graph theory related literature, different approaches and techniques are proposed for balanced graph partitioning.

\subsection{Existing Graph Partitioning Techniques}

In [13], a fast approximate graph-partitioning algorithm 
is proposed. The authors unified the problems of $b$-balanced cuts and $k$-multiway separators using a new approach called minimum capacity $\rho$-separators. They studied the graph partitioning problems on graphs with edge capacities and vertex weights and described a simple approximation algorithm for minimum capacity $\rho$-separators leading to a fast approximation algorithm both for $b$-balanced cuts and $k$-multiway separators. They define a $\rho$-separator as a sub-set of edges whose removal partitions the vertex set into connected components such that the sum of the vertex weights in each component is at most $\rho$ times the weight of the graph.

In [14], authors considered three problems to find a $(l$, $u)$-partition of a given graph. They proposed to partition a graph $G$ into connected components by deleting some edges from $G$ making the total weight of each component equal at least to $l$ and at most to $u$. The minimum partition problem is to find an $(l, u)$-partition with the minimum number of components, the maximum partition problem is defined in the same way and the p-partition problem is to find an $(l, u)$-partition with a fixed number $p$ of components. Authors proved that the three problems are NP-complete or NP-hard.

In [15], authors studied the approximation of the Maximally Balanced Connected Partition problem (MBCP). They first presented the optimization problem that finds the maximally balanced connected partition for a graph $G$. It results in a partition $\left(V_{1}, V_{2}\right)$ of $V$ composed of disjoint sets $V_{1}$ and $V_{2}$ such that both sub-graphs of $G$ induced by $V_{1}$ and $V_{2}$ are connected, and maximize an objective function "balance", $B^{w}\left(V_{1}, V_{2}\right)=\min \left(w\left(V_{1}\right)\right.$, $\left.w\left(V_{2}\right)\right)$. Authors proved that the problem is NP-hard.

In this work, this last approach will be adapted and applied to our Network. Our choice is mainly motivated by the practical approach provided in [15] and based on the use of a polynomial-time algorithm that gives an approximate solution.

In the following the Maximally Balanced Connected Partition (MBCP) technique [15] is adapted and formulated. A corresponding approximate resolution algorithm is then presented.

\subsection{Problem Formulation}

Since, in Base Stations placement scheme, we are considering the communication between the Cluster Heads and the Base Stations (the upper tier of the architecture), only Cluster Heads are concerned by the partitioning scheme. We assume then that if a Cluster Head belongs to a sub-network, then its corresponding Cluster belongs to this sub-network as well.

We consider here the undirected connected graph $G(H, A)$. We remind that $H$ is the set of Cluster Heads, $H=\left\{\mathrm{CH}_{i}, i=1\right.$ to $\left.N\right\}$ and $A$ the set of all undirected links.
The objective is to partition $G$ into connected balanced sub-graphs.

To achieve this objective, let $w$ be a non-negative vertex-weight function representing the balancing criteria. In this case, $w$ will reflect the number of Cluster Heads. Hence $w\left(H^{\prime}\right)=\left|H^{\prime}\right|$.

This MBCP problem can then be formulated as follow:

Maximize $B^{w}\left(H_{1}, H_{2}\right)=\min \left(w\left(H_{1}\right), w\left(H_{2}\right)\right)$

Subject to $1 .\left(H_{1}, H_{2}\right)$ is a partition of $H$ into nonempty disjoints sets $H_{1}$ and $H_{2}$ such that sub-graphs of $G$ induced by $H_{1}$ and $H_{2}$ are connected.

$$
\text { 2. } w(H \mathrm{n})=\sum_{C H_{i} \in H \mathrm{n}} w\left(C H_{i}\right) \quad \forall \quad H \mathrm{n} \subseteq H
$$

The resolution of this model will result into two balanced sub-networks. Each of them can be partitioned again using the same process.

This partitioning technique should be applied as much as required according to the targeted size for the subnetworks and taking into account the number of available Base Stations to be placed. The final result should be $2^{\text {n }}$ equivalent smaller connected sub-networks where $n$ is the number of partitioning iterations.

\subsection{Problem Resolution}

To solve this model, we used the polynomial approximation algorithm presented in [15] and which finds an approximate solution for the MBCP problem.

In order to select neighbouring Cluster Heads within the same sub-networks, we adapted this algorithm by sorting the list of candidates for each partition according to their distance (vicinity). Figure 3 illustrates an example of a WSN partitioned into four sub-networks.

The algorithm can be written as follow:

Input: $G=(H, A)$.

$\mathrm{H}=\left\{\mathrm{CH}_{1}, \mathrm{CH}_{2}, \mathrm{CH}_{3} \ldots \mathrm{CH}_{\mathrm{N}}\right\}$ where $\mathrm{N}=|\mathrm{H}|$.

1) Initialize $H_{1}=\left\{\mathrm{CH}_{1}\right\}, \mathrm{H}_{2}=H \backslash H_{1}$ such as $\mathrm{CH}_{1}$ a Cluster Head near the periphery of the network.

2) If $\left|H_{1}\right|>=1 / 2|H|$ then Step 3 else Step 2.

3) Let $H_{0}=\left\{\mathrm{CH}_{i} \in \mathrm{H}\left(\mathrm{H}_{1} U\left\{\mathrm{CH}_{i}\right\}, \mathrm{H}_{2} \mid\left\{\mathrm{CH}_{i}\right\}, i \in\right.\right.$ $\{1 . . N\})$ is a connected partition of $G\}$.

Choose $\mathrm{CH}_{i}$ of $\mathrm{H}_{0}$ such that $\mathrm{CH}_{i}$ the closest element to $H_{1}$.

If $\left|C H_{i}\right|<|H|-2\left|H_{1}\right|$

then $H_{1}:=H_{1} U\left\{\mathrm{CH}_{i}\right\}, H_{2}:=H_{2} \mid\left\{C H_{i}\right\}$, Step 1

else Step 3

4) Return $\left(H_{1}, H_{2}\right)$.

\subsection{Locating Base Stations}

Once the network is partitioned into identical smaller 


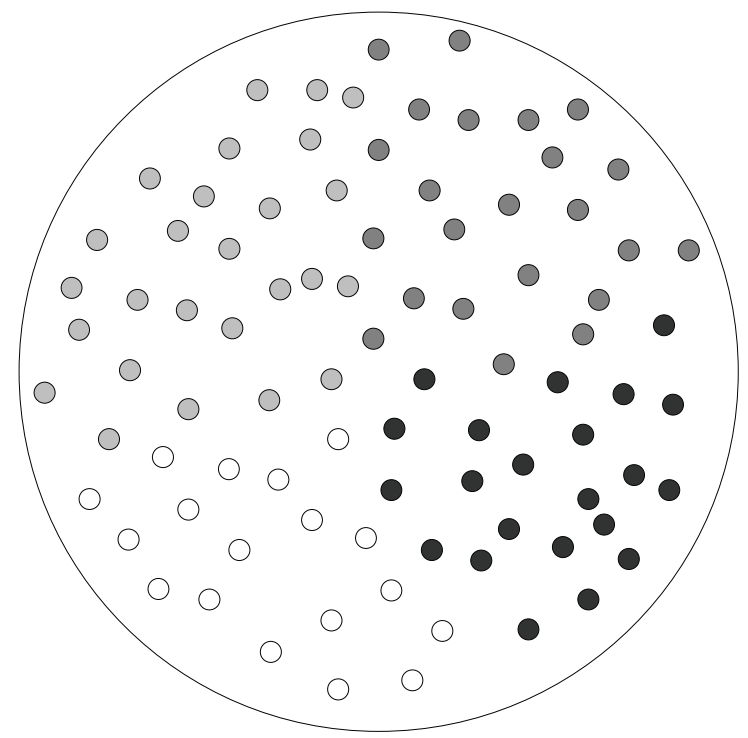

Figure 3. A Wireless Sensor Network partitioned into 4 sub-networks (the four node patterns represent four different sub-networks).

two-tiered sub-networks, each of these sub-networks is represented by a disc with the geographic centre of the sub-network as centre and the distance between this centre and the farthest Cluster Head (belonging to this subnetwork) from it as radius. Recall that if a Cluster Head belongs to a sub-network, then its Cluster members belong to this sub-network as well.

Base Stations can now be optimally located within each of these sub-networks independently but in the same way.

It has been proven in [8] that the optimum movement for a mobile base station is to follow the periphery when the deployment area is circular.

Motivated by this result, we suggest in this work that one single Base Station be randomly deployed on the periphery of each sub-network. Then each Base Station keeps moving along the periphery of the sub-network in which it was deployed. Note that Cluster Heads can send their data only to the Base Station deployed in the sub-network they belong to.

A mobile base station can move in two different regimes, a fast mobility regime and a slow mobility regime [16]. In the fast mobility regime, the base station moves in a continuous form with a velocity $v$ along the time without any stop or pause in a particular position. In the slow mobility regime, the base station moves in a discrete form and its trajectory is a sequence of anchor points between which the base station moves with a velocity $v$ and at which it pauses during a period of time (epoch).

The slow mobility regime is considered more realistic and is adopted in many research studies. Therefore, we assume in this work that each Base Station moves in a slow mobility regime. We propose then to divide time into rounds. Each Base Station moves then at the beginning of each round and remains at its new position until the end of the round.

It is very important to carefully choose the value of the base station velocity. In fact, when the mobile base station velocity is high, the base station will more frequently change its position and visit more the different regions over the area of interest during the network lifetime. Therefore, the energy consumption is efficiently distributed over the cluster heads and the network lifetime is extended. This can be much more efficient in the particular case where the cluster heads buffer the data gathered in their clusters and wait until the base station approaches to deliver it [4] which reduces unnecessarily packet forwarding actions since cluster heads are sure of base station arrival before loosing the data (because of buffer size limitation or packet deadline expiration). Besides, the high speed moving base station produces a tolerable data delivery delay especially in the case of fast mobility regime, which can be very important for some specific applications. However, base station high velocity can have negative effects. In fact, it can make the session interval too short to successfully exchange a long data packet and hence the packet loss rate will increase. In slow mobility regime, it is preferred that the epoch (round) be long enough to guaranty long messages exchange.

On the other hand, it seems obvious that the mobility of the base stations will inevitably incur additional overhead in data exchanges since the cluster heads will continuously need to be informed of their corresponding base station location. However, it has been proved in [16] that when using a slow mobility regime with an epoch much longer than the base station moving time, the overhead introduced by the mobility of the base station became negligible because amortized across a long epoch. This reinforces our choice in using a slow mobility regime.

After determining the Base Stations placement strategy, we can further prolong network lifetime by instructing Cluster Heads to efficiently forward the data to the destination. Hence, at the beginning of each round and after it is located in its new position, each Base Station has to compute the routing scheme that will manage in an energy efficient manner the inter Cluster Heads communication within its corresponding sub-network

\section{Inter-Cluster Head Communication}

As discussed at the beginning of this paper, Cluster Heads that are in critical positions run out of energy first. Hence, to further extend the network lifetime, it is necessary to delay as much as possible the first Cluster Heads death. 
For small-scale non-clustered WSNs, we proposed in a previous work [5] an approach that defines an optimal multi-hop routing. It dynamically distributes flows proportionally to the residual energy available at each node leading to a maximum network lifetime.

The routing scheme is modelled as an optimization algorithm and is computed at the Base Station. Its resolution results in a routing matrix that defines for each node to which of its neighbors it has to send data.

In this section, we propose to extend this approach to two-tiered WSN architectures. In addition to the residual energy at each Cluster Heads, we introduce a new constraint that reflects Cluster Head energy consumption related to its intra-cluster activities (i.e. the first role of Cluster Heads). The idea is to alleviate, from relaying activities (i.e. the second role of Cluster Heads), Cluster Heads requiring higher energy for managing their clusters.

On the other hand, inside each cluster, Sensing Nodes have to provide the information required by the end application. They should be organized such that the QoS is satisfied with minimum cost. Different techniques can be used to achieve this goal. For instance, sensors can be autonomous and self organized [6,17]. Another approach is to use a relative central mechanism (e.g., scheduling mechanism) that can take the appropriate decisions on behalf of the Sensing Nodes. For instance, we can consider that within each cluster, one or more Sensing Nodes may be used at any time to provide data to the application, but only certain subsets of available sensors may satisfy channel bandwidth and/or application quality of service constraints [18]. In this work, we decide to adapt the scheduling mechanism, initially proposed in [18] for a flat topological WSNs, to manage communications inside the clusters. This scheduler determines which sensor sets should be used and for how long time so that the lifetime of the cluster is maximized while the necessary quality of service expected from this cluster is always maintained at the application. In addition, Sensing Nodes providing redundant information can be turned off which contributes in energy saving and reduces data flows. Used within each cluster and according to the performance evaluation given in [18], this mechanism optimizes individual clusters lifetimes.

In order to achieve a global routing optimization, the inter-Cluster Heads communication approach that we propose should, in addition, take into account these individual clusters lifetimes, as the more a cluster lasts, the more its Cluster Heads requires energy for its management (e.g., reception, data processing and fusion, ...).

This inter-Cluster Heads communication approach is modeled within each sub-network as an optimization problem. It is then processed in a centralized manner at the Base Station of each sub-network independently but simultaneously. It takes into account the current status and topology of the sub-network and results in a routing matrix that defines the inter-Cluster Heads flows within this sub-network such that the minimum Cluster Head lifetime is optimized.

The inter-Cluster Heads communication approach construction and its details are presented in the following sections.

\subsection{Model and Notations}

Let's consider $N_{b}$ Base Stations to be deployed in the network. We note a Base Station $k$ by $b_{k}, k=1$ to $N_{b}$. The network graph $G$ is then partitioned into $N_{b}$ equivalent sub-graphs. We consider $\left(H_{1}, H_{2}, \ldots, H_{N_{b}}\right)$ the connected partition of $G$.

Then, each sub-network $k$ corresponding to $H_{k}$ contains one single mobile Base Station $b_{k}$ and $N_{k}^{C H}$ Cluster Heads, $k=1$ to $N_{b}, \sum_{k} N_{k}^{C H}=N$.

We assume that each sub-network $k$ is modeled as a connected sub-graph $G_{k}\left(H_{k}, A_{k}\right), k=1$ to $N_{b} . H_{k}$ is then the set of Cluster Heads belonging to the sub-network $k$, $H_{k}=\left\{C H_{k, i}, i=1\right.$ to $\left.N_{k}^{C H}\right\}$ and $A_{k}$ the set of the undirected links $\left(\mathrm{CH}_{k, i}, \mathrm{CH}_{k, j}\right)$ where $\mathrm{CH}_{k, i}$ and $\mathrm{CH}_{k, j}$ are two Cluster Heads of $H_{k}$.

Let $L_{k, i}$ be the set of Cluster Heads neighbors of Cluster Head $\mathrm{CH}_{k, i}$ in the sub-network $k . L_{k, i}$ is composed of all Cluster Heads of $H_{k}$ that can be reached by $\mathrm{CH}_{k, i}$. All links are assumed to be bidirectional.

We remind that if a Cluster Head belongs to a sub-network than its corresponding Cluster belongs to this sub-network as well. We will note by $C_{k, i}$ the Cluster of Sensing Nodes corresponding to the Cluster Head $\mathrm{CH}_{k, i}$ and then belonging to sub-network $k, i=1$ to $N_{k}^{C H}$ and $k=1$ to $N_{b}$.

Each cluster $C_{k, i}$ contains $N_{k, i}^{S}$ Sensing Nodes. We will refer to the complete set of Sensing Nodes within a cluster $C_{k, i}$ as $S_{k, i}=\left\{S_{k, i l}, l \in\left\{1, \ldots, N_{k, i}^{S}\right\}\right\}$.

We remind that all Sensing Nodes in Cluster $C_{k, i}$ can communicate directly with their Cluster Head $\mathrm{CH}_{k, i}$ and that all Cluster Heads $\mathrm{CH}_{k, i}$ belonging to sub-network $k$ have to forward the gathered data to the Base Station $b_{k}$ deployed within this same sub-network. Also, Cluster Heads belonging to one sub-network cannot communicate with Cluster Heads belonging to another sub-network.

We finally assume that $E_{k, i l}^{S}$ and $E_{k, i}^{C H}$ are the initial energies of Sensing Node $S_{k, i l}$ and Cluster Head $\mathrm{CH}_{k, I}$ respectively. In Table 1, we list all symbols used in the rest of this paper.

\subsection{Flow Conservation}

We denote by $r_{k, i}$ the arrival rate of information at $\mathrm{CH}_{k, I}$ 
Table 1. Notations.

\begin{tabular}{|c|c|}
\hline Symbol & Description \\
\hline$N$ & the number of Cluster Heads/Clusters in the network. \\
\hline$H$ & the set of $N$ Cluster Heads of the WSN. \\
\hline$A$ & $\begin{array}{l}\text { the set of the undirected links between the Cluster } \\
\text { Heads of } H\end{array}$ \\
\hline $\mathrm{CH}_{i}$ & a Cluster Head of $H$. \\
\hline$C_{i}$ & the Cluster corresponding to $\mathrm{CH}_{i}$. \\
\hline$L_{i}$ & the set of Cluster Heads neighbours of $\mathrm{CH}_{i}$. \\
\hline$N_{b}$ & the number of base stations deployed in the network. \\
\hline$H_{k}$ & a partition of $H$. \\
\hline$b_{k}$ & the base station deployed in sub-graph $k$. \\
\hline $\mathrm{CH}_{k, i}$ & a Cluster Head of $H_{k}$. \\
\hline$N_{k}^{C H}$ & the number of Cluster Heads in sub-graph $k$. \\
\hline$L_{k, i}$ & $\begin{array}{l}\text { the set of Cluster Heads Neighbors of } \mathrm{CH}_{k, i} \text { in } \\
\text { sub-graph } k \text {. }\end{array}$ \\
\hline$C_{k, i}$ & the cluster in sub-network $k$ corresponding to $\mathrm{CH}_{k, i}$. \\
\hline$N_{k, i}^{S}$ & the number of Sensing nodes in $C_{k, i}$. \\
\hline$S_{k, i}$ & the set of Sensing Nodes in $C_{k, i}$. \\
\hline$S_{k, i l}$ & a Sensing Node of $S_{k, i}$. \\
\hline$E_{k, i l}^{S}$ & the initial energy of $S_{k, i l}$. \\
\hline$E_{k, i}^{C H}$ & the initial energy of $\mathrm{CH}_{k, i}$. \\
\hline$r_{k, i}$ & the arrival rate of sensed data at $\mathrm{CH}_{k, i}$. \\
\hline$v_{k, i}$ & the arrival rate of aggregated data at $\mathrm{CH}_{k, i}$. \\
\hline$\beta$ & the data agregation ratio. \\
\hline$f_{a}$ & the aggregation function. \\
\hline$w_{k, i}$ & the average rate of data that transit through $\mathrm{CH}_{k, i}$. \\
\hline$w_{b_{k}}$ & the average rate of data that transit through $b_{k}$. \\
\hline$p_{k, i j}$ & The flow portion transmitted from $\mathrm{CH}_{k, i} \mathrm{CH}_{k, j}$. \\
\hline$P_{k}$ & the routing matrix within sub-network $k$. \\
\hline$T_{k, i}^{C}$ & the lifetime duration of $C_{k, i}$. \\
\hline$T_{k, i}^{C H}$ & the lifetime duration of $\mathrm{CH}_{k, i}$. \\
\hline$T_{k}$ & the lifetime duration of sub-network $k$. \\
\hline$T_{n e t}$ & the lifetime duration of the whole network. \\
\hline$F_{k, i}$ & the set of feasible sensor sets in $C_{k, i}$. \\
\hline$F_{k, i m}$ & a feasible sensor set of $F_{k, i}$. \\
\hline$N_{k, i}^{F}$ & the number of feasible sensor sets in $C_{k, i}$. \\
\hline$T_{k, i m}^{F}$ & $\begin{array}{l}\text { the length of time that } F_{k, i m} \text { is being used in the optimal } \\
\text { Schedule of } C_{k, i} \text {. }\end{array}$ \\
\hline$q_{k, i l}$ & the power consumption at sensor $S_{k, i l}$. \\
\hline$E_{\text {elec }}$ & the energy consumed to run the radio electronics. \\
\hline$\varepsilon_{a m p}$ & the energy consumed to run the power amplifier. \\
\hline$e_{k, i j}$ & $\begin{array}{l}\text { the transmission energy required to transmit one data } \\
\text { unit from } \mathrm{CH}_{k, i} \text { to } \mathrm{CH}_{k, j} \text {. }\end{array}$ \\
\hline$e_{r}$ & the energy required for the reception of one data unit. \\
\hline$e_{a}$ & the energy required to the fusion of one data unit. \\
\hline$\gamma$ & the aggregation energy consumption coefficient. \\
\hline
\end{tabular}

sensed by the Sensing Nodes within its cluster $C_{k, i}$ and we denote by $v_{k, i}$ the rate of information at $\mathrm{CH}_{k, i}$ after aggregation.

Hence, $v_{k, i}$ can be written as, $v_{k, i}=f_{a}\left(r_{k, i}\right) . f_{a}$ is a typical linear aggregation function such that $f_{a}(x)=\beta x$ for some constant $\beta, 0<\beta<1 . \quad \beta$ is called the data aggregation ratio [1].

Let $w_{k, i}$ be the average rate of information that transit through $\mathrm{CH}_{k, i}$. It is composed of the generated information rate at $\mathrm{CH}_{k, i}$ (sensed by the cluster members and then aggregated at $\mathrm{CH}_{k, i}$ ) plus the information rate received from its Cluster Heads neighbours of $L_{k, i}$.

$w_{k, i}$ is given by:

$$
w_{k, i}=v_{k, i}+\sum_{j / C H_{k, j} \in L_{k, i}} p_{k, j i} w_{k, j} \quad \forall\left(k, i \in\left\{1, \ldots, N_{k}^{C H}\right\}\right)
$$

and

$$
w_{b_{k}}=\sum_{i \in\left\{1, \ldots, N_{k}^{C H}\right\}} v_{k, i}
$$

where $p_{k, j i} w_{k, j}$ is the proportion of data transmitted by $\mathrm{CH}_{k, j}$ to $\mathrm{CH}_{k, i}$.

Obviously, $p_{k, i j} \geq 0 \forall(k, i, j)$ and $\sum_{j / C H_{k, j} \in L_{k, i}} p_{k, i j}$ $=1 \forall\left(k, i \in\left\{1, \ldots, N_{k}^{C H}\right\}\right)$.

We denote by $P_{k}$ the routing matrix within subnetwork $k$ and which can be written as:

$$
P_{k}=\left\{p_{k, i j}\right\}
$$

Note that Equations (4) and (5) verify the flow conservation condition. The flow conservation condition states that the sum of information generation rate and the total incoming flow must equal the total outgoing flow.

\subsection{Lifetime Model}

We remind that a cluster dies when no more reliable information can be delivered from the cluster Sensing Nodes. We denote the lifetime of a cluster $C_{k, i}$ by $T_{k, i}^{C}$. Once its cluster dead, each Cluster head continue performing relaying activities until it is over of energy. We then denote by $T_{k, i}^{C H}$, the lifetime of Cluster Head $\mathrm{CH}_{k, i}$.

The lifetime of the whole network is defined, as stated in Subsection 3.4, as the period of time that ends when a first Cluster Head runs out of energy. We analogically define the lifetime of a sub-network $k$ as the period of time until which the first Cluster Head $\mathrm{CH}_{k, i}$ dies and denote it by $T_{k}$. Then, $T_{k}$ can be written as:

$$
T_{k}=\min _{i \in\left\{1, \ldots, N_{k}^{C H}\right\}} T_{k, i}^{C H}, \quad \forall k
$$


Thus, the network lifetime can be defined as the period of time until which the first sub-network dies.

The network lifetime, denoted by $T_{n e t}$, can then be written as follow:

$$
T_{n e t}=\min _{k} T_{k}=\min _{k, i \in\left\{1, \ldots, N_{k}^{C H}\right\}} T_{k, i}^{C H}
$$

Hence, maximizing the network lifetime can be achieved by maximizing each sub-network lifetime simultaneously.

\subsection{Intra-Cluster Communication}

As already mentioned, the intra-cluster communication scheme is inspired from [18]. The communications inside the clusters is managed by an optimized scheduler that determines which sensor sets should be used and for how long time so that the lifetime of the cluster is maximized while the necessary quality of service is respected.

As defined in [18], a sensor set is determined to be feasible if 1) the total bandwidth necessary to support the set is below the capacity of the cluster and the traffic is schedulable and 2) the set provides the necessary reliability to the application. We will refer to the set of feasible sensor sets in a cluster $C_{k, i}$ as $F_{k, i}=\left\{F_{k, i m}, m \in\left\{1, \ldots, N_{k, i}^{F}\right\}\right\}$.

The optimal scheduler that maximizes the lifetime of $C_{k, i}$ determines the length of time that each sensor set in $C_{k, i}$ should be used. Let $T_{k, i m}^{F}$ represent the length of time that feasible sensor set $F_{k, i m}$ is being used in the optimal schedule of $C_{k, i}$. The objective of the problem is to maximize the lifetime of each cluster $C_{k, i}$ :

$$
T_{k, i}^{C}=\sum_{m} T_{k, i m}^{F} \forall\left(k, i \in\left\{1, \ldots, N_{k}^{C H}\right\}\right)
$$

We will define $a_{k, i l m}$ as a variable equal to one if sensor $S_{k, i l}$ is being used in feasible sensor set $F_{k, i m}$ of the cluster $C_{k, i}$ and equal to zero otherwise.

Finally, we define $q_{k, i l}$ as a variable that represents the power consumption (sensing and communication) at sensor $S_{k, i l}$.

We remind that $E_{k, i l}^{S}$ is the initial energy of Sensor Node $S_{k, i l}$. This finite energy introduces the following constraint:

$$
\sum_{m} a_{k, i l m} T_{k, i m}^{F} q_{k, i l} \leq E_{k, i l}^{S} \forall\left(k, i \in\left\{1, \ldots, N_{k}^{C H}\right\}, l \in\left\{1, \ldots, N_{k, i}^{S}\right\}\right)
$$

This scheduling problem has been modeled as a generalized maximum flow graph problem. The same method will be used for each cluster in the network and carried out in a centralized manner by an unconstrained node or at the application level at the beginning of the network deployment and once the clusters are formed (during the set-up phase and before the transmission phase is started). The computation of this optimization scheme defines for each cluster the optimal Schedule that maximizes its lifetime. Each Cluster lifetime value can then be computed and used as an input parameter for the inter-Cluster Heads communication scheme.

To have details about the resolution of this optimization problem the reader is referred to [18].

\subsection{Maximizing Network Lifetime}

According to the scheduling problem described in the last section the lifetime of each cluster $C_{k, i}$ (not including the corresponding $\left.\mathrm{CH}_{k, i}\right)$ is $T_{k, i}^{C}$. During this period of time a Cluster Head $\mathrm{CH}_{k, i}$ is providing two functionalities: the first concerns internal exchange (receiving and aggregating data coming from its cluster members) and the second concerns external exchange (receiving, transmitting and relaying the data coming from its Cluser Head neighbors).

Once this period achieved, $\mathrm{CH}_{k, i}$, if not yet drained out of energy, expend its remaining energy to provide only the second functionality.

During the period of time $T_{k, i}^{C}, C H_{k, i}$ expends an amount of energy given by Equation (10).

Here, $e_{k, i j}$ is the transmission energy required to transmit one data unit from $\mathrm{CH}_{k, i}$ to $\mathrm{CH}_{k, j}$ relatively to Equation (1).

So, the remaining energy at $\mathrm{CH}_{k, i}$ when $T_{k, i}^{C}$ is spent is:

$$
E_{k, i}^{C H_{2}}=E_{k, i}^{C H}-E_{k, i}^{C H_{1}}
$$

Hence, according to the energy model described in Subsection 3.3, the lifetime of $\mathrm{CH}_{k, i}$ under a given system $P_{k}=\left\{p_{k, i j}\right\} \forall\left(k, i \in\left\{1, \ldots, N_{k}^{C H}\right\}\right)$ is given by Equation (12).

$$
\begin{gathered}
E_{k, i}^{C H_{1}}=T_{k, i}^{C}\left(\sum_{j / C H_{k, j} \in L_{k, i}} e_{k, i j} p_{k, i j} w_{k, i}+\sum_{j / C H_{k, j} \in L_{k, i}} e_{r} p_{k, j i} w_{k, j}+\left(e_{a}+e_{r}\right) r_{k, i}\right) \\
T_{k, i}^{C H}\left(P_{k}\right)=T_{k, i}^{C}+\frac{E_{k, i}^{C H}}{\sum_{j / C H_{k, j} \in L_{k, i}} e_{k, i j} p_{k, i j} w_{k, i}+\sum_{j / C H_{k, j} \in L_{k, i}} e_{r} p_{k, j i} w_{k, j}} \\
=T_{k, i}^{C}+\frac{E_{k, i}^{C H}-T_{k, i}^{C}\left(\sum_{j / C H_{k, j} \in L_{k, i}} e_{k, i j} p_{k, i j} w_{k, i}+\sum_{j / C H_{k, j} \in L_{k, i}} e_{r} p_{k, j i} w_{k, j}+\left(e_{a}+e_{r}\right) r_{k, i}\right)}{\sum_{j / C H_{k, j} \in L_{k, i}} e_{k, i j} p_{k, i j} w_{k, i}+\sum_{j / C H_{k, j} \in L_{k, i}} e_{r} p_{k, j i} w_{k, j}}
\end{gathered}
$$


Then, $T_{k}$, the lifetime of sub-graph $k$, can be approximated as follow:

$$
T_{k}\left(P_{k}\right)=\min _{i \in\left\{1, \ldots, N_{k}^{C H}\right\}} T_{k, i}^{C H}\left(P_{k}\right), \forall k
$$

Maximizing the lifetime of a sub-network $k$ can be reached by solving the following optimization problem:

$$
\begin{array}{ll}
\text { Maximize } & T_{k} \\
\text { Subject to } & p_{k, i j} \geq 0 \forall i \in\left\{1, \ldots, N_{k}^{C H}\right\} \text { and } \forall j / C H_{k, j} \in L_{k, i} \\
& \sum_{j / C H_{k, j} \in L_{k, i}} p_{k, i j}=0 \forall i \in\left\{1, \ldots, N_{k}^{C H}\right\} \\
& E_{k, i}^{C H_{1}}+E_{k, i}^{C H_{2}} \leq E_{k, i}^{C H} \quad \forall i \in\left\{1, \ldots, N_{k}^{C H}\right\}
\end{array}
$$

The last constraint models energy conservation at each Cluster Head $\mathrm{CH}_{k, i}$.

The resolution of this system requires determining the matrix $P_{k}$ defining, for a fixed position of Base Station $b_{k}$, the optimal routing flows that are used by each Cluster Head within sub-network $k$ to forward data to its Neighbors such that the lifetime of this sub-network is maximized. The optimal matrix $P_{k}$ can then be computed in a centralized fashion at the Base Station $b_{k}$.

This optimisation problem is Non Polynomial and can then be solved over Matlab using specific heuristics similar to those used to solve the optimization problem presented in [5]. Once the different sub-networks lifetimes $T_{k}, \forall k=1$ to $N_{b}$ are computed, the whole network lifetime can be finally given by:

$$
T_{\text {net }}=\min _{k} T_{k}
$$

\section{Global Framework}

In this section we describe the overall dynamic framework for large two-tiered wireless sensor networks lifetime maximization. The framework is based on the optimisation scheme related to both Base Stations positioning and inter-Cluster Head communication presented previously. A cyclic algorithm is then defined to permit the dynamic adaptation of the optimization process (see Figure 4).

Once the nodes are deployed in the interested area, the network topology is first abstracted and the overall network is partitioned into equivalent sub-networks that have the same characteristics and where the energy consumption can be optimized independently but in the same way. One mobile base station is then randomly deployed on the periphery of each sub-network. Time is then divided into equal periods of time called rounds or epochs. At the beginning of each round, each base station moves along the periphery of its corresponding subnetwork. Once it reached its new position, the base station collects information about the current topology
Input: $G(H, A)$.

0.1. The network is divided into $N_{b}$ equivalent sub-networks.

0.2. One mobile base station is deployed on the periphery of each of these sub-networks.

0.3. Initial round duration (epoch) is determined at the application level

While (the sensor network is operational for the application) do $\{/ /$ begin of the round

$$
\forall k \in\left\{1, \ldots, N_{b}\right\}:
$$

1) Base station $b_{k}$ in sub-network $k$ moves to its new position on the periphery

2) At base station $b_{k}$ : Collection of all relevant information from all the cluster heads of $\mathrm{H}_{\mathrm{k}}$ concerning the current topology of sub-network $k$.

3) At base station $b_{k}$ : Run of the optimization process and compute the routing matrix $\left[\mathrm{P}_{\mathrm{k}}\right]$.

4) Base station $b_{k}$ transmits to each Cluster Head $\mathrm{CH}_{k, i}$ the vector $\left[\mathrm{P}_{\mathrm{k}, \mathrm{ij}}\right]$

$$
\left(\forall i \in\left\{1 \ldots N_{k}^{C H}\right\} \text { and } \forall j / C H_{k, j} \in L_{k, i}\right) .
$$

5) Each Cluster Head sends the captured/received information to its neighbors toward $b_{k}$ according to $\left[\mathrm{P}_{\mathrm{k}}\right]$.

// end of the round

\section{Figure 4. Global framework.}

status of its sub-network. These information may include The residual energy at each sensor node, the neighbors list and the positions of each node, sources' throughputs, etc.

In a next step, each base station runs the routing optimization process corresponding to its sub-network as described in the previous section and which results in an updated routing matrix that optimally distributes energy consumption over the different Cluster Heads according to their roles in the sub-network and to the residual amount energy at each of them. Data gathering is then performed by the sensing nodes and the collected data is aggregated and forwarded by the cluster heads toward the corresponding base station using the optimized routing probabilities.

\section{Simulations}

This section is dedicated to the evaluation of the performances of first, the Base Stations Placement scheme that optimally locates the different base stations in the network while considering scalability as well as energy efficiency issues and second, the inter-Cluster Head communication approach formulated as an optimization problem that aims to efficiently and fairly distribute the energy among Cluster Heads while taking into account their roles in the network.

\subsection{Base Stations Placement}

The effect of the proposed partitioning technique on the WSN lifetime is investigated using numerical simulations over Matlab environment. A circular large-scale wireless sensor network, with a radius $\mathrm{R}=500 \mathrm{~m}$ is con- 
sidered. In order to study the performance of the base stations placement scheme, we focused on the upper tier of the network architecture (Base Stations and Cluster Heads) independently of the lower tier (Cluster Heads and Sensing Nodes). 1000 nodes (Cluster Heads) are randomly (uniformly) deployed over a network area. All nodes are similar with a communication range $r=80 \mathrm{~m}$ and an initial energy of $1000 \mathrm{~J}$ unit. Base Stations are assumed to have no energy constraints because they have larger batteries or their batteries are rechargeable. We assumed, in this scenario, that the shortest path routing algorithm is used to establish routes from Cluster Heads to base stations. The network lifetime is defined as the moment at which the first node runs out of energy. Time is divided into rounds. Each round is composed of $T=$ 100 timeframes. Each sensor node generates one data packet every timeframe.

To evaluate the efficiency of the proposed graph partitioning technique in elongating the network lifetime, three comparative scenarios are considered:

1) Scenario 1:

Case 1: An entire large network (not partitioned) is considered. All the sensors have the same capacity. $\mathrm{N}$ base stations are randomly fixed inside the coverage area of interest. Each sensor has to send the data it senses to the nearest base station.

Case 2: The graph-partitioning algorithm (detailed in Subsection 4.3) is used to define N smaller sub-networks. One single base station is then randomly fixed in each sub network. Each sensor node sends its data to the base station deployed inside the sub-network the sensor node is belonging to.

2) Scenario 2:

Case 1: The entire network is considered. $\mathrm{N}$ mobile base stations are deployed randomly. Then, the base stations start to move inside the area of interest following the random waypoint model [19]. At the beginning of each round, each base station moves $60 \mathrm{~m}$.

Case 2: $\mathrm{N}$ sub-networks are defined using the graphpartitioning algorithm and one single base station is randomly deployed in each sub network. Then each base station moves $60 \mathrm{~m}$ each round. The base station cannot go outside the area of the sub-network it belongs to. This area is represented by a disc with the geographic centre of the sub-network as centre and the distance between this centre and the farthest sensor (belonging to this subnetwork) from it as radius.

3) Scenario 3:

Case 1: The entire network is considered. $\mathrm{N}$ mobile base stations are deployed randomly on the periphery of the network. Then, the base stations start to move along the periphery. In one round each base station moved 60 $\mathrm{m}$.

Case 2: The graph-partitioning algorithm is used to define $\mathrm{N}$ smaller sub-networks. One single base station is randomly deployed on the periphery of each sub network.
Then each base station moves $60 \mathrm{~m}$ each round on the periphery.

We consider that the time required by a base station to move to its next position is negligible compared to a round duration.

Several simulations are then run to compare the network lifetime in the two different cases of each of the three different scenarios.

Simulation results are presented in Figures 5, 6 and 7.

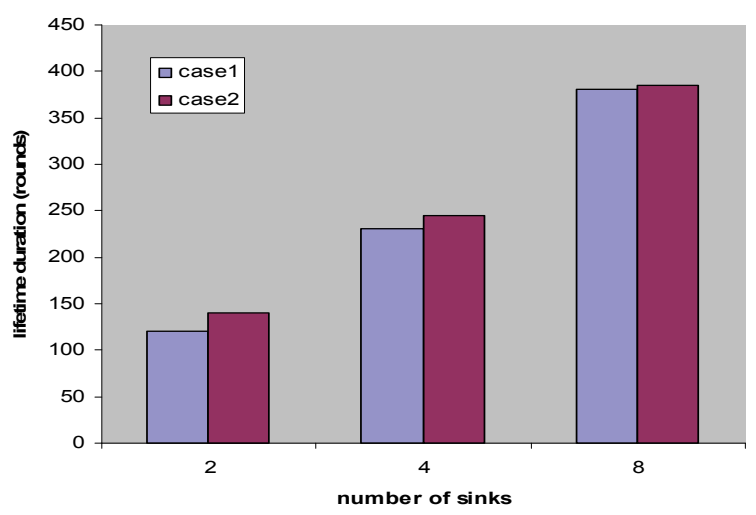

Figure 5. The network lifetime in Scenario 1.

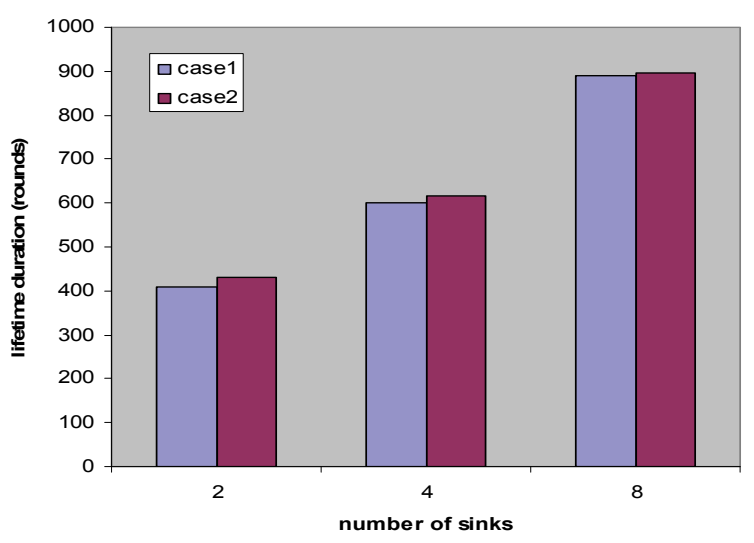

Figure 6. The network lifetime in Scenario 2.

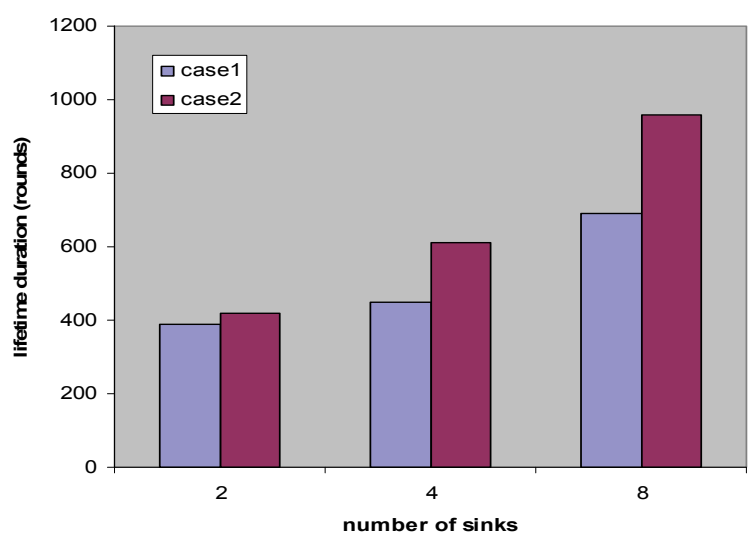

Figure 7. The network lifetime in Scenario 3. 
They respectively compare the performance of the different base stations deployment strategies in the case of partitioned and non-partitioned network (Scenario 1, 2 and 3 ).

First, let's notice that the simple use of multiple base stations enhances the network lifetime (with and without partitioning). Indeed, the network lifetime increases proportionally to the number of base stations because the distance between the nodes and their correspondent base stations is shortened. Second, it can be seen that moving the base stations clearly prolong the operation of the network. In fact, figures show that the network lifetime is much longer when the base stations are moving (Scenario 2 and 3 with or without partitioning) than when they are fix (Scenario1). This result is valid with or without partitioning.

Third, enhancements of the network lifetime can be observed in the case of partitioned large-scale WSNs compared to non-partitioned ones in all the scenarios. But the enhancement is the most significant in the third scenario. This was expected as when one base station is moving along the periphery of each sub-network, the energy consumption is obviously much more distributed over the sensors than when all the base stations are moving along the periphery of the whole network. The nodes that are the closest to the base stations are logically the ones who die first because they not only send their own data but also relay the data of all the nodes in the network. In Scenario 3, the nodes who die first in the case of non-partitioned network are the nodes situated all along the periphery whereas in the case of partitioned network, they are the ones situated along the peripheries of the different sub-networks. Then, in this scenario, using the graph partitioning technique to deploy the base stations distributes the load relay and decreases the average distance between the nodes and the base stations. Indeed, the improvement of the network lifetime of the partitioned network is much more important when the number of base stations (or sub-networks) increases.

In the first case of the first scenario, base stations are randomly placed. Hence, they can be in some cases grouped in a small space. As a consequence, the distance between a node and the closest base station may not be really shortened. Whereas, in the second case, where we limited the area in which each base station can be deployed, by partitioning the network into sub networks, this distance is almost always shortened. This can be much more efficient when the base stations move (Scenario 2) since the base stations in both cases have the same velocity (60 m/round).

However, we notice, from Figure 5 and Figure 6, that the improvement is not so spectacular. This can be explained by the fact that when dividing the network into independent sub-networks, some nodes are bound to send their data to the base station deployed in the sub-network they belong to whereas they are closer to a base station deployed outside (in an other sub-network).

\subsection{Inter-Cluster Heads Communication}

In this section, we focus on the performance evaluation of the optimization scheme presented in section 5 and which manages the communication between Cluster Heads whithin each sub-network to efficiently transmit data toward base stations. The optimization problem is solved using specific heuristics and several simulations were run over Matlab.

Since the same optimal routing process is used in each of the sub-networks, we limit here our simulations to one single sub-network. We consider then a circular subnetwork with radius equal to $100 \mathrm{~m}$. Cluster Heads and Sensing nodes are assumed to have a maximum communication radius of $80 \mathrm{~m}$ and $20 \mathrm{~m}$ respectively. We assume that nodes are, initially, distributed in a random fashion over the sub-area and that the clusterization is based on neighborhood. Feasibles sets are then randomly generated in each cluster of the sub-network. One base station with no energy constraints is deployed and randomly placed on the periphery of the area.

The same initial energy is assumed for all Cluster Heads and is equal to $1000 \mathrm{~J}$ unit. The same initial energy is also assumed for all Sensing Nodes and is equal to $50 \mathrm{~J}$. Power consumption at the Sensing Nodes is 10 $\mu \mathrm{W}$.

The following values are considered for energy dissipation at Cluster Heads.

$E_{\text {elec }}=50 \mathrm{~nJ} / \mathrm{bit}$ in the transmit circuitry and

$\epsilon_{\text {amp }}=100 \mathrm{pJ} / \mathrm{bit} / \mathrm{m}^{2}$ for the transmit amplifier.

$\gamma=50 \mathrm{~nJ} / \mathrm{bit}$ for the aggregation energy consumption.

We assume the data aggregation ratio $\beta=25 \%$ and a Sensing Node data rate equal to $160 \mathrm{bit} / \mathrm{s}$.

Figures are obtained by averaging simulation results for a large number of scenarios. For each scenario, a different random node layout is used.

Figure 8 illustrates the normalized sub-network lifetime. As depicted, the numerical resolution of the proposed model quickly converges to an optimal solution.

To study the effect of the sub-network composition and topology on its lifetime and the interactions between the inter-cluster and intra-cluster communications, we study the scenario where the sizes of the clusters vary while the number of cluster heads is kept constant. When running the simulations, we randomly generate feasible sets for each cluster. The number of feasible sets in a cluster is randomly chosen. The number of cluster heads is fixed at 20. Initially, we randomly generate the number of sensing nodes in each cluster while keeping the average number equal to 3 . Then, we increase the number of sensing nodes similarly in each cluster until it reaches 18 (average size).

The results are presented in Figure 9, which illustrates 


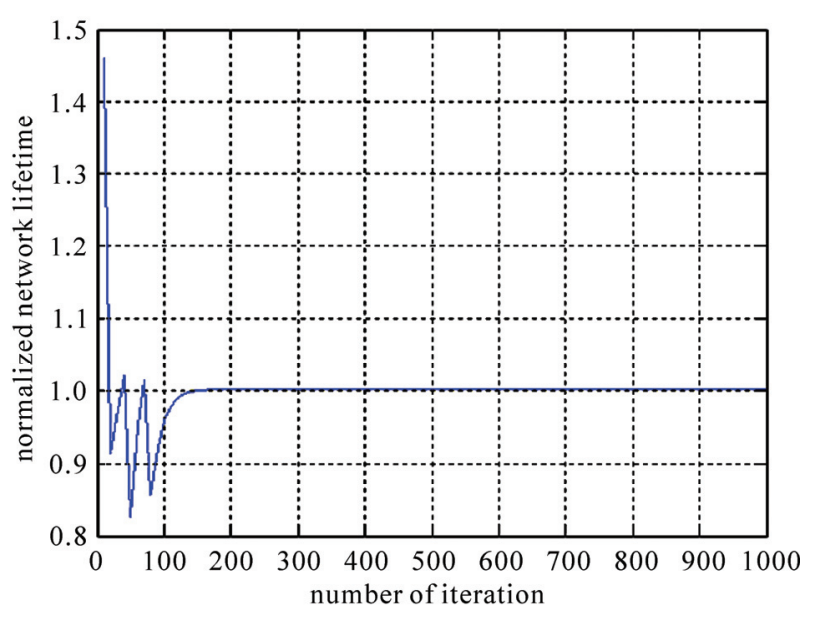

Figure 8. Lifetime convergence.

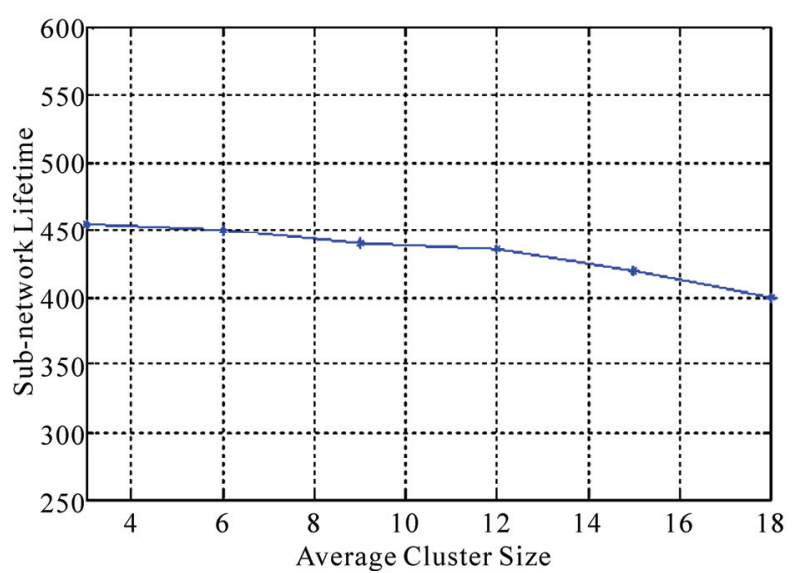

Figure 9. Sub-network lifetime as a function of the clusters size.

a sub-network lifetime evolution when increasing the clusters' size and keeping the number of cluster heads constant.

It can be seen that the sub-network lifetime decreases as the clusters size increases. This is expected as when the cluster size increases, the corresponding cluster lifetime increases as well. Hence, each cluster head will spend more time performing both its neighbor's data relay and its own cluster management (its two roles simultaneously). As a result, it expends more quickly its energy which leads to network death in shorter time.

To further explore the performances of the proposed inter-cluster head communication scheme, we propose to study the influence of the clusters lifetime on the choice of the routes to deliver the data from each Cluster Head to the base station. An efficient routing scheme should alleviate from releying tasks cluster heads with long clusters lifetime since these cluster heads will spend longer time and then much more energy to manage their clusters than those with short cluster lifetime. To this end, we voluntarily generate clusters with considerably dif- ferent lifetimes (through different sizes). This makes the corresponding clusters' lifetime standard deviation be large.

After several simulations, we compute the different cluster head lifetime and we remark that the corresponding standard deviation is considerably small $(3.2 \%$ of the whole sub-network lifetime). This result proves that the majority of cluster heads die approximately at the same time. This also proves that flows are fairly distributed over the different cluster heads proportionally to the residual energy available at each one of them and also with considering the lifetime of each cluster i.e., proportionally to their role in the sub-network. The objectives of the proposed schemes are obviously attained.

\section{Conclusions}

The use of multiple mobile base stations in large-scale wireless sensor networks is necessary in order to cover large areas and to minimize energy consumption for data transmission operations. In this paper, we proposed an energy efficient usage of multiple, mobile base stations to increase the lifetime of a two-tiered large-scale Wireless Sensor Network. Our approach uses a graph-partitioning algorithm to decompose the underlying network into balanced sub-networks. The energy usage is then optimized in each sub-network independently but in the same way using efficient base stations placement techniques that are optimized for small-scale WSNs. Performance results have shown that the proposed technique considerably enhances the network lifetime particularly when the base stations are moving along the periphery.

We have further proposed an optimal multi-hop routing scheme used within each sub-network independently to efficiently manage the communication between the Cluster Heads so that the entire network lifetime is elongated. Different strategies can be used, inside clusters, to manage intra-cluster communications. The proposed scheme simply adapt and fairly distribute the relaying flows according to Cluster Heads residual energy and their corresponding Clusters' lifetime duration, so that Cluster Heads with critical energy situations are alleviated from relaying operations. Simulation results have shown that we can compute a near optimal solution of the routing matrix that defines the optimal flow routing.

The overall dynamic framework that combines the above two schemes has been then described. It is defined as a cyclic algorithm that allows dynamic adaptation of the optimization process according to the current status of the whole network.

Using the graph-partitioning approach to improve energy consumption in large-scale WSNs is promising. We will focus in complementary and future work on more elaborated approaches for optimal multiple mobile base stations placement and WSN partitioning. In addition, 
efficient tools should be proposed to determine the optimal number of partitions and base stations to be used according to the WSN characteristics, applications' requirements and financial costs.

Moreover, we plan in future work to investigate further the mathematical resolution of the optimization algorithm corresponding to the inter-Cluster Head communication. The effect on energy consumption of the overhead generated by this scheme needs to be more deeply explored.

\section{References}

[1] Y. P. Chen, A. L. Liestman and J. Liu, "A Hierarchical Energy-Efficient Framework for Data Aggregation in Wireless Sensor Networks," IEEE Transactions on Vehicular Technology, Vol. 55, No. 3, May 2006, pp. 789796.

[2] W. Rabiner Heinzelman, A. Chandrakasan and H. Balakrishnan, "Energy-Efficient Communication Protocol for Wireless Microsensor Networks," Proceedings of the 33rd International Conference on System Sciences, Hawaii, January 2000, pp. 3005-3014.

[3] J. Pan, Y. Hou, L. Cai, Y. Shi and X. Shen, "Topology Control for Wireless Sensor Networks," Proceeding of the 9th ACM Conference on Mobile Computing and Networking, San Diego, September 2003, pp. 286-299.

[4] C. Chen, J. Ma and K. Yu, "Designing Energy-Efficient Wireless Sensor Networks with Mobile Sinks," Proceeding of ACM Sensys'06 Workshop WSW, Boulder, October 2006, pp. 1-9.

[5] I.Slama, B. Jouaber and D. Zeghlache, "Routing for Wireless Sensor Networks Lifetime Maximization under Energy Constraints," The 3rd International Conference on Mobile Technology, Applications and Systems, Bangkok, October 2006, pp. 1-5.

[6] W. Heinzelman, A. Chandrakasan and H. Balakrishnan, "An Application-Specific Protocol Architecture for Wireless Microsensor Networks," IEEE Transaction in Wireless Communications, Vol. 1, No. 4, October 2002, pp. 660-670.

[7] V. Mhatre, C. Rosenberg, D. Kofman, R. Mazumdar and N. Shroff, "A Minimum Cost Heterogeneous Sensor Network with a Lifetime Constraint," IEEE Transaction on Mobile Computing, Vol. 4, No. 1, 2005, pp. 4-15.

[8] J. Luo and J.-P. Hubaux, "Joint Mobility and Routing for Lifetime Elongation in Wireless Sensor Networks," Pro- ceeding of IEEE INFOCOM, Miami, March 2005, pp. $1-10$.

[9] Z. M. Wang, S. Basagni, E. Melachrinoudis and C. Petrioli, "Exploiting Sink Mobility for Maximizing Sensor Networks Lifetime," Proceedings of the 38th Annual Hawaii International Conference on System Sciences, Washington, D.C., January 2005, p. 287.

[10] S. R. Gandham, M. Dawande, R. Prakash and S. Venkatesan, "Energy Efficient Schemes for Wireless Sensor Networks With Multiple Mobile Base Stations," Proceeding of IEEE GLOBECOM, San Francisco, 2003, pp. 377-381.

[11] H. Kim, Y. Seok, N. Choi, Y. Choi and T. Kwon, "Optimal Multi-Sink Positioning and Energy-Efficient Routing in Wireless Sensor Networks," Lecture Notes in Computer Science, Vol. 3391, No. 11, pp. 264-274.

[12] Z. Vincze, K. Fodor, R. Vida and A. Vidacs, "Electrostatic Modelling of Multiple Mobile Sinks in Wireless Sensor Networks," Proceeding of IFIP Networking Workshop on Performance Control in Wireless Sensor Networks, Coimbra, May 2006, pp. 30-37.

[13] G. Even, J. Naor, S. Rao and B. Schieber, "Fast Approximate Graph Partitioning Algorithms," Proceeding of the 8th Annual ACM-SIAM Symposium on Discrete Algorithms, New Orleans, 1997, pp. 639-648.

[14] T. Ito, X. Zhou and T. Nishizeki, "Partitioning a Graph of Bounded Tree-Width to Connected Subgraphs of Almost Uniform Size," Journal of Discrete Algorithms, Vo. 4, No. 1, March 2006, pp. 142-154.

[15] J. Chlebikova, "Approximability of the Maximally Balanced Connected Partition Problem in Graphs," Information Processing Letters, Vol. 60, 1996, pp. 225-230.

[16] J. Luo, J. Panchard, M. Piorkowski, M. Grosglausser and J-P. Hubaux, "Mobiroute: Routing towards a Mobile Sink for Improving Lifetime in Sensor Networks", Proceeding of the International Conference on Distributed Computing in Sensor Systems, San Francisco, June 2006.

[17] M. Chatterjee, S. K. Das and D. Turgut, "WCA: A Weighted Clustering Algorithm for Mobile Ad Hoc Networks," Journal of Cluster Computing, Special Issue on Mobile Ad Hoc Networking, Vol. 5, No. 2, 2002, pp. 193-204.

[18] M. Perillo and W. Heinzelman, "Optimal Sensor Management Under Energy and Reliability Constraints," Proceedings of the IEEE Wireless Communications and Networking Conference, New Orleans, March 2003.

[19] D. B. Johnson and D. A. Maltz, "Dynamic Source Routing in Ad Hoc Wireless Networks," Mobile Computing, Vol. 353, No. 5, pp. 153-181. 